Cahiers $d u$ MONDE RUSSE

\section{Cahiers du monde russe}

Russie - Empire russe - Union soviétique et États indépendants

50/2-3 | 2009

L'Europe orientale, 1650-1730. Crises, conflits et renouveau

\title{
To the Tashkent StationRebecca MANLEY
}

, Ithaca - Londres : Cornell University Press, xvi+282 p.

\section{Juliette Denis}

\section{(2) OpenEdition}

\section{Journals}

Édition électronique

URL : https://journals.openedition.org/monderusse/9786

DOI : $10.4000 /$ monderusse.9786

ISSN : $1777-5388$

Éditeur

Éditions de l'EHESS

Édition imprimée

Date de publication : 15 septembre 2009

ISBN : 978-2-7132-2260-3

ISSN : $1252-6576$

Référence électronique

Juliette Denis, "To the Tashkent StationRebecca MANLEY », Cahiers du monde russe [En ligne], 50/2-3 | 2009, mis en ligne le 14 janvier 2013, consulté le 03 septembre 2022. URL : http:// journals.openedition.org/monderusse/9786; DOI : https://doi.org/10.4000/monderusse.9786

Ce document a été généré automatiquement le 3 septembre 2022

Tous droits réservés 


\section{To the Tashkent StationRebecca MANLEY}

, Ithaca - Londres : Cornell University Press, xvi+282 p.

Juliette Denis

\section{Rebecca MANLEY, To the Tashkent Station, Evacuation and Survival in the Soviet Union at War, Ithaca - Londres : Cornell University Press, $x v i+282$ p.}

1 L'historiographie de la Seconde Guerre mondiale et de la sortie de guerre en URSS est depuis quelques années en plein renouvellement. La thèse de Rebecca Manley s'inscrit pleinement dans cette dynamique. L'auteur étudie la gigantesque migration, provoquée par le conflit mondial, d'un groupe d'individus définis tant par les conditions de guerre que par leur déplacement en URSS : les évacués. Ces seize millions et demi de citoyens soviétiques issus de huit républiques constituent une catégorie spécifique partiellement dépendante d'une initiative étatique. La structure du livre reflète une histoiretrajectoire : les étapes des évacuations se succèdent au gré du déplacement du front, des décisions de Moscou et des hasards du chaos de la Grande Guerre patriotique. De ce déplacement de populations, Rebecca Manley propose une analyse inédite, à la croisée de l'histoire stalinienne, de celle de l'URSS et de l'Europe en guerre.

2 L'auteur reprend avec finesse l'histoire de l'évacuation en URSS. Pratique fréquente dans l'historiographie récente de la Seconde Guerre mondiale, elle rappelle avec pertinence le précédent de la Première, où la question des civils et de leur déplacement se pose pour la première fois dans un contexte international. La notion même d'évacuation apparaît dans l'Empire tsariste en 1915 par le biais d'une commission spécifiquement créée à cet effet. Témoignage d'un changement de conception de la guerre totale, ce terme réservé jusqu'alors au domaine militaire - désigne désormais une opération de grande envergure de déplacement des ressources économiques et humaines. Le pouvoir bolchevique s'appuie sur cette expérience pour organiser les flux de populations au moment de la guerre civile. Il distingue le «déplacement volontaire», reposant à ses 
yeux sur une décision individuelle (même si elle est suscitée par les contraintes du front), des expulsions imposées d'en haut, à des fins militaires ou autres. Un décret en 1937, puis le brouillon d'un décret (finalement non enteriné) en 1938, placent l'évacuation dans le double cadre de la gestion des déplacements de populations en URSS et de la nouvelle perception de la guerre moderne: sous le choc de Guernica et de la violence des bombardements, la planification intègre enfants ainsi que catégories inactives et élargit les territoires concernés par l'évacuation. Le terme de réfugié disparait progressivement des documents officiels. Mais, malgré les réflexions entreprises dès les années 1930, l'évacuation n'entre pas dans la conception personnelle de la guerre offensive propre à Stalin. Aussi l'invasion de 22 juin 1941 oblige-t-elle à repenser ce concept en urgence. Le 24 juin, un Conseil de l'évacuation est créé, qui procède à une opération gigantesque, mais sélective. La définition des catégories à évacuer, puis celle de leurs conditions de transport et d'existence dans les profondeurs de l'URSS, reflète les hiérarchies de la société soviétique, qui repose sur une élite jeune, urbaine, intégrée à l'armée, au parti et à l'État, ou qui occupe une position dominante dans les arts et les sciences. Ces privilégiés sont les évacués officiels, à distinguer des "auto-évacués » fuyant sans autorisation et dans des conditions souvent catastrophiques.

3 L'une des thématiques les plus passionnantes de l'ouvrage est la place de l'évacuation dans l'histoire des déplacements forcés soviétiques. Les années 1930 ont vu s'accroître aussi bien la volonté de contrôle du mouvement des citoyens soviétiques que les vagues d'exil forcé de communautés entières, à travers la passeportisation de 1932 et les déplacements forcés vers les colonies spéciales. Dans le déroulement de l'évacuation, un rôle crucial est donné au NKVD, organe qui par ailleurs avait acquis un savoir-faire nécessaire en matière logistique. Évacuation et déportation procèdent d'une même vision globale des déplacements de population. L'ambiguïté est évidente dès lors que l'on touche à la question des minorités frontalières: les Allemands de Crimée ou de Leningrad passent par exemple, selon les décrets et rapports, du statut de colons spéciaux à celui d'évacués.

4 À l'échelle locale, on observe cependant un décalage entre planification et pratiques, qu'explique un contexte de peur, de panique, de fuite massive et prématurée des représentants de l'État et d'« auto-évacuation » de la population. Le désordre est à son comble à Minsk, l'une des premières villes à tomber aux mains des Allemands, contrairement à Odessa où une préparation plus longue a assuré le succès de l'opération. À Leningrad, l'organisme mis en place pour évacuer les civils permet aux catégories les plus vulnérables d'échapper au blocus; à Moscou l'évacuation s'accélère à l'automne 1941. Par ailleurs, l'évacuation reflète aussi l'absence de consensus au sein de la société soviétique : certains ne veulent pas partir. Le "should I stay or should I go " soviétique est rarement simple désir de survivre. Le dilemme est forgé par des conceptions personnelles du devoir et de la loyauté; selon les groupes, l'évacuation est perçue comme une opération de secours ou comme une expulsion. Le prisme de l'évacuation permet de cerner les contours poreux de la signification du patriotisme soviétique en temps de guerre, et, d'un point de vue méthodologique, de nuancer la séparation nette entre motivations personnelles et devoir civique.

5 Après un trajet plus ou moins confortable et dangereux selon le lieu de départ, la position sociale, l'avancée du front, la durée des transits et les attentes dans les gares, les évacués arrivent à destination. Bien qu'apparue tardivement dans les plans de relocalisation, Tachkent est une destination privilégiée : elle est considérée comme une 
ville importante, russophone, réputée pour son climat, son exotisme, les possibilités de ravitaillement. Cependant, la réalité est moins reluisante : les évacués commencent leur nouvelle vie dans une ville perçue comme "étrangère ", et surtout dans des conditions de vie, en temps de guerre, particulièrement difficiles. Ils suscitent la méfiance des autorités et du NKVD, qui les soupçonnent d'être des spéculateurs, des " personnes de l'ombre ", des espions, en particulier si leur départ n'a pas été officiellement reconnu. La propiska indispensable n'est pas toujours delivrée et des familles séparées par les circonstances de la guerre ont tout le mal du monde à obtenir les papiers nécessaires à leur regroupement. Dans la crainte constante des contrôles de police, ces personnes sont contraintes, pour survivre, à l'illégalité ou au système D. Fin 1941, on compte 100000 évacués à Tachkent. Leur nombre augmente l'année suivante: la ville se remplit d'écrivains, d'artistes, d'usines, d'universités... Se nourrir, se loger deviennent des préoccupations permanentes, dont la résolution dépend de la position dans la société soviétique, de l'officialité de l'évacuation, et surtout de réseaux sociaux ou familiaux d'entraide. Femmes et enfants, surreprésentés, sont les populations les plus vulnérables, mais la mortalité est importante pour tous. Le retour dans un lieu fantasmé, et dont les évacués finissent par rêver, s'avère également ardu : comme à l'heure de l'évacuation, les autorisations ne sont pas systématiquent accordées. Retrouver une place souvent précaire dans la société d'après-guerre est également tâche ardue. Face aux héros et aux victimes de la guerre, les évacués se sentent stigmatisés par un parcours en marge de la collectivité en guerre.

Les ambiguités de l'évacuation, à tous les stades de son déroulement, sont au cœur de la démonstration de Rebecca Manley. Ainsi en Ouzbékistan, les évacués n'échappent pas à la réalité de la guerre: ils participent au "front du travail », ou au "front culturel » (l'exemple d'Anna Ahmatova est le plus connu). Mais l'expression « front de Tachkent ", popularisée dans l'URSS en guerre, finit par stigmatiser les lâches et les déserteurs. Cette accusation est plus particulièrement dirigée contre les évacués d'origine juive, surreprésentés dans les contingents d'évacués, bien qu'aucune politique n'ait été mise en œuvre pour les sauver des atrocités nazies. L'évacuation, et plus encore le mouvement du retour, sont concomitants de la montée de l'antisémitisme dans la société soviétique. Insultes, incidents, voire agressions, vécus au moment du départ ou à Tachkent les poursuivent jusqu'à leur retour; sauvés par l'évacuation, les évacués rentrent néanmoins dans des terres « sans juifs », mais surtout stigmatisés comme juifs.

Certaines questions restent en suspens, avant tout celle des sources qui ne font pas l'objet d'une présentation pourtant attendue. L'analyse du volume ou de la localisation des archives aurait permis d'appréhender les charges respectives attribuées au pouvoir central et aux régions d'accueil dans la gestion des évacués. Journaux intimes et mémoires de guerre, sollicités presque exclusivement dans certains chapitres, laissent parfois supposer que les archives administratives sont absentes ou inaccessibles. Avec une seule carte, l'ouvrage ne propose qu'une visualisation géographique très limitée des parcours, alors que la diversité des situations aurait pu susciter une typologie et une cartographie de la panique, des zones d'évacuation prioritaires, et de quelques trajectoires personnelles suivies du début à la fin de la guerre. Quid des expériences spécifiques, qui auraient pu permettre d'approfondir les ambivalences entre évacuation et répression; quel est le destin des prisonniers évacués, ou des Polonais en Asie centrale, ces déportés fraîchement amnistiés qui ont dû s'intégrer à la société soviétique? Comment sont accueillis les réfugiés juifs qui arrivent illégalement dans les territoires 
soviétiques? Autant d'interrogations qui feront certainement l'objet de recherches futures.

8 Ces critiques n'entachent en rien l'excellence de cette étude, qui, multipliant les échelles d'analyse de l'État à la trajectoire personnelle, donne à voir la complexité et l'ampleur de l'évacuation. En conclusion, le travail de Rebecca Manley propose, au-delà de sa thématique spécifique, une vision de l'histoire soviétique fondée sur l'étude des déplacements de populations et de leur contrôle, contribuant à renouveler la perception de l'URSS stalinienne en guerre. 